\title{
Coupling a Gas Chromatography Unit to an Inductively Coupled Plasma Mass Spectrometer
}

\author{
Jerrad P. Auxier ${ }^{1,2}$, John D. Auxier II ${ }^{1,2,3}$, Howard L. Hall1,2,3 \\ ${ }^{1}$ Department of Nuclear and Radiological Engineering, University of Tennessee, Knoxville, TN, USA \\ ${ }^{2}$ Radiochemistry Center of Excellence (RCOE), University of Tennessee, Knoxville, TN, USA \\ ${ }^{3}$ Center for Public Policy, Institute for Nuclear Security, University of Tennessee, Knoxville, TN, USA \\ Email: jauxier1@vols.utk.edu
}

How to cite this paper: Auxier, J.P., Auxier II, J.D. and Hall, H.L. (2017) Coupling a Gas Chromatography Unit to an Inductively Coupled Plasma Mass Spectrometer. World Journal of Nuclear Science and Technology, 7, 84-92.

http://doi.org/10.4236/wjnst.2017.72007

Received: January 27, 2017

Accepted: April 16, 2017

Published: April 20, 2017

Copyright (C) 2017 by authors and Scientific Research Publishing Inc. This work is licensed under the Creative Commons Attribution International License (CC BY 4.0).

http://creativecommons.org/licenses/by/4.0/

\begin{abstract}
Although the eminent threat of a terrorist group detonating an improvised nuclear device (IND) in an urban environment is low, it is crucial that countries develop modern nuclear forensic capabilities to expedite response in a post-detonation scenario. In particular, new instruments need to be created to shorten dissolution time, expedite chemical separation, and improve forensic analysis of the nuclear melt glass that is created during the detonation of the device. To expedite this process, an instrument was designed to thermally couple a gas chromatograph (GC) to a time-of-flight inductively coupled plasma time-of-flight mass spectrometer (ICPTOFMS) In order to couple these two instruments, another instrument was designed to provide an isothermal atmosphere between the GC and TOFICPMS to expedite rapid gas separations processes. By using gas separations instead of the current wet chemistry processes, the required separation and analysis time of the melt glass significantly decreases. The new instrument would also provide a more detailed analysis of the elemental and isotopic composition of the melt glass. By completing these tasks simultaneously, this significantly decreases the required time to conduct these separations and improves the elemental and isotopic analysis.
\end{abstract}

\section{Keywords}

Nuclear Forensics, Gas Chromatography, Mass Spectrometry, Melt Glass, Chemical Separation, Nuclear Security, Elemental Analysis

\section{Introduction}

The threat of a nuclear weapon detonation by a terrorist organization is a concern to the NNSA and other government agencies that are tasked with the secu- 
rity of the nation and its citizens. These agencies must create novel methods for safeguarding special nuclear material and developing post-denotation forensic analysis techniques. In the event that an improvised nuclear device is detonated the debris that is generated contains valuable elemental and isotropic data to help identify the age and processing location of the nuclear material. To obtain this information wet chemistry analysis techniques are used; however, these methods for investigating post-detonation debris and materials are tedious and prolonged. The purpose of this research is to develop novel chemical separation methods to shorten analysis time and improve the retrieved data from the debris. To expedite the analysis of the fall out debris, a new instrument for gas phase separation will be discussed.

A method of coupling a traditional gas chromatograph to an inductively coupled plasma time of flight mass spectrometer was investigated. The gas chromatography (GC) system will be used to begin the separation process by heating sample material to the gas phase. The samples eluted from the GC will be transported to the mass spectrometer (MS) for analysis through a column encased in a temperature controlled coupling unit. Using this combination of instrument, separations of post-event debris can be performed while simultaneously acquiring elemental and isotopic signatures in the same experiment. Initial experiments have shown that this apparatus can improve separation times from the standard $\sim 7 \mathrm{hr}$. to $<1 \mathrm{hr}$.

\section{Theory}

Wet chemistry processes are currently used to separate and prepare melt glass samples created during a nuclear blast for analysis. The basic processes include dissolution, precipitation, solvent extraction, ion exchange, and sample analysis. These methods successfully achieve elemental separation and preparation; the process requires long periods of time and material to accurately characterize the melt glass [1].

To decrease the analysis time and material requirements, the melt glass can be separated and analyzed while it is in the gas phase, thereby eliminating several of the sample preparation processes. The method explored herein begins after sample dissolution of post detonation samples. The samples are taken from the liquid phase to the gas phase and analyzed via thermo-chromatography techniques that can be performed accurately and timely. To perform this process, a gas chromatography unit is used to separate chemically modified debris samples [2] [3]. The gas mixture is carried through a quartz column and the compounds separate based on thermodynamic parameters as described by Auxier et al. and Eichler et al. [4] [5]. This process can be modeled by a Monte Carlo model that utilizes the different enthalpies of absorption and desorption that occurs between the lanthanide compounds and the wall of the column. The lanthanoid compounds are heavier than the carrier gas and contain different properties with regard to enthalpy and entropy. The lanthanide compounds will adsorb to the sides of the quartz column and depending on the characteristics of the individual 
elements they will desorb from the column thus causing them to separate into individual peaks, see Figure 1. Based on the differences in entropy and enthalpy of adsorption and desorption between the compounds and the quartz column, the elemental compounds can be separated. In order to obtain the necessary elemental and isotopic analysis, the gas chromatography unit is coupled to an inductively coupled plasma time-of-flight mass spectrometer (ICP-TOF-MS). The ICP-TOF-MS is capable of delivering the required isotopic and element data such that the elemental ratios of the post detonation sample can be calculated.

To ensure accurate and reproducible results, the adsorption, desorption, and flow velocity must be controlled. The adsorption and desorption terms are related by temperature and is calculated in Equation (1) where $\tau_{a}$ is the adsorption of the column, $\tau_{0}$ is the oscillatory period of the molecule, $\Delta H_{A}$ is the adsorption enthalpy, $R$ is the gas constant, and $T$ is the temperature of the column. The gas flow through the column is defined in Equation (2), where $F_{m}$ is the reference flow of gas at the beginning of the column, $T_{C}$ is the column temperature, $T$ is the temperature of the gas at the flow meter, $P$ is the gas pressure at the end of the column, and $P_{\mathrm{H}_{2} \mathrm{O}}$ is the correction factor for the pressure in case the solution is saturated by water.

$$
\begin{gathered}
\tau_{a}=\tau_{0} * \mathrm{e}^{-\left(\frac{\Delta H_{A}}{R T}\right)} \\
F=F_{m} *\left(\frac{T_{C}}{T}\right) *\left(\frac{P-P_{\mathrm{H}_{2} \mathrm{O}}}{P}\right)
\end{gathered}
$$

In previous work with super-heavy elements (SHEs) [6] [7], the column was lined with alpha and beta particle detectors, however for the purposes of this work, the column is coupled to the ICP source of the MS system. To couple the quartz column between these two instruments, a heat transport apparatus was built that would connect the GC unit to the MS and provide a temperature controlled environment for the quartz column.

According to the analysis addressed by Garrison et al. and Hanson et al. [8], the temperature of the column drastically changes the results of the chromatography. Therefore it is crucial to analyze the thermophysical properties of the

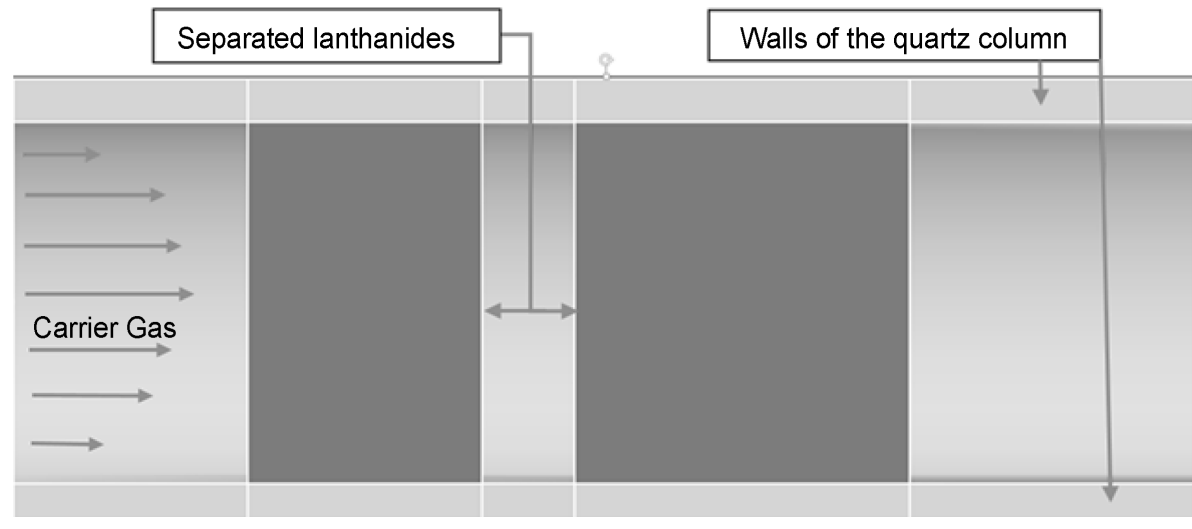

Figure 1. Separation that occurs in the column of the GC. 
column to ensure that the proposed apparatus design will create an isothermal atmosphere.

The apparatus was designed to encapsulate the column in an atmosphere of air; according Schimdt et al. [9], the material properties between the contents in the column and the heat exchanger material should be similar. If the material properties are similar, the efficiency of the convective heat transfer rate remains high which causes the heat transfer heat rate to remain turbulent. As the air cools around the column, the difference in air temperature between the column and face of the casing causes the hot air to circulate around the column; this creates an isothermal atmosphere around the column. The following equations are used to calculate weather the heat transfer rate is turbulent, and how to calculate the convective heat transfer rate.

Turbulent convective heat transfer occurs when the value of the Reynolds number (Re) exceeds 2300, and this value can be calculated by Equation (3). Where $\rho_{g}$ is the density of the gas inside the column, $V_{g}$ is the velocity of the gas inside the column, $d_{c}$ is the diameter of the column, and $\mu_{g}$ is the dynamic viscosity of the gas

$$
\operatorname{Re}=\frac{\rho_{g} * V_{G} * d_{c}}{\mu_{g}}
$$

Once the transition between laminar and turbulent flows occurs, the value of the Nusselt number can be calculated by using Equation (4). Where Re is the Reynold's Number calculated in Equation (3), Pr is the Prandtl number of the quartz column, $d_{h}$ is the hydraulic diameter of the column, and $L$ is the length of the column. After this average value of the Nusselt number is calculated, a correction factor must be applied to ensure accuracy, see Equation (5); where $(N U)$ is the average Nusselt number, $T_{m}$ is the temperature of the gas inside the column, and $T_{w}$ is the temperature of the gas surrounding the column and $N U_{\text {cor }}$ is the corrected Nusselt number.

$$
\begin{gathered}
\overline{N U}=0.0214\left(\operatorname{Re}^{\frac{4}{5}}-100\right) *(\operatorname{Pr})^{\frac{2}{5}} *\left[1+\left(\frac{d_{h}}{L}\right)^{\frac{2}{3}}\right] \\
N U_{\text {cor }}=\overline{N U} *\left(\frac{T_{m}}{T_{w}}\right)^{N}
\end{gathered}
$$

The corrected Nusselt number is then used in Equation (6) to determine the final convective heat transfer coefficient, which measures if a turbulent convective heat is maintained. Where $N U_{\text {cor }}$ is the corrected Nusselt number, $k$ is the thermal conductivity of the material, and $d_{c}$ is the diameter of the column.

$$
\bar{h}=\frac{N u_{\text {cor }} * k}{d}
$$

\section{Building New Instrument}

Because of the high temperatures required for accurate radiochemical separations, the material used to construct the apparatus must withstand temperatures 
greater than $450^{\circ} \mathrm{C}$ [10]. Therefore the heaters, thermocouples, and structural materials were selected to tolerate these temperatures. The structural material that was selected was a stainless steel 316 alloy with a melting temperature exceeding $1000 \mathrm{~K}$.

According to the previously shown equations, the adsorption and desorption properties are directly related to the change in temperature in the column [11]. To maintain a uniform isothermal environment for the column, the case surrounding the column must be heated uniformly. A series of multiple, independently controlled, heating elements was chosen to provide the necessary uniformity [12]. The heaters are required to operate independently to be able to control the thermal dynamics of the case depending on the location of the heater on the pipe. The heaters at the ends of the case/pipe will operate more to account for the thermal loss due to the lack of insulation [13]. To account for the thermal changes and to provide the required thermal stability, six Wattco heaters were connected to the casing surrounding the column. The six different heaters attached to the casing ensures that the heat supplied to the sides of the casing is even and that the temperature of the column remains constant $\left( \pm 2^{\circ} \mathrm{C}\right)$.

After the heaters were attached to the sides of the column, thermocouples were attached in specific positions along the column. According to the calculations previously discussed, to accurately control the temperature along the column, there needs to be no less than three heating zones along the length of the pipe. To maintain the required isothermal atmosphere, there were four thermocouples attached to the casing around the pipe column to control and monitor the heating zones. The rate of heat loss is the largest at the ends of the apparatus, therefore the thermocouples at these locations control two of heaters instead of only one heater.

The thermocouples (Figure 2) used are Omega, Cement On, C01-E thermocouples. The C01-E were chosen because of their fast response time and low thermal inertia. These thermocouples are recommended for attachment to either flat or curved materials that are composed of metal, plastic, or ceramic surfaces. The C01-E style is made from a thermocouple alloy foil that is fabricated such

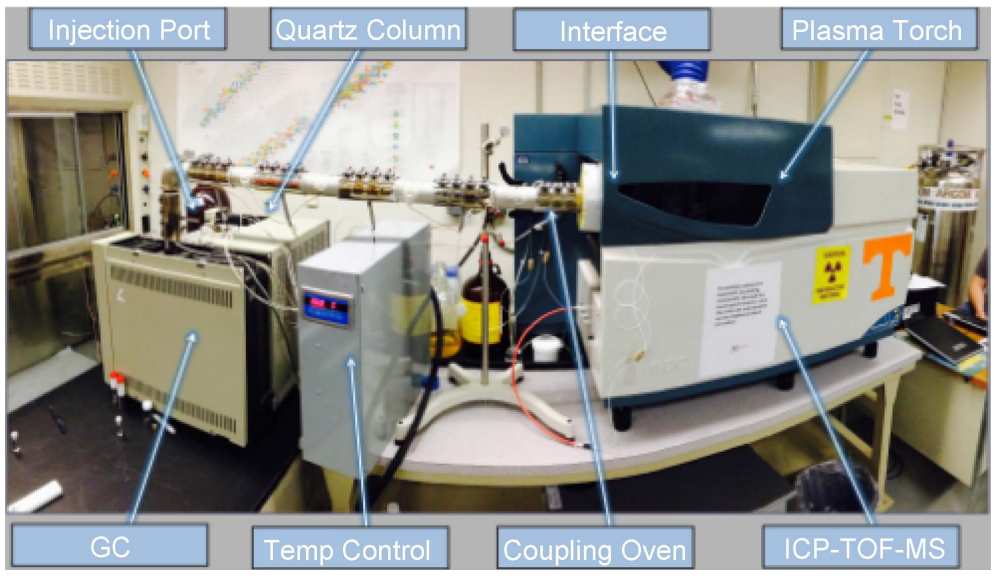

Figure 2. The completed instrument. 
that the contacts of the thermocouple are only $0.013 \mathrm{~mm}$ thick; this ensures that the thermocouple detects small changes in temperature within a small period of time. The C01-E thermocouples were fabricated according to the specifications of American National Standards Institute (ANSI). Special Limits of Error material specifications with regard to the error margin they allow when measuring the temperature. Once the C01-E thermocouples were attached to the casing, they were calibrated through the controller by using a laser thermometer to ensure the accurate temperature was measured.

The thermocouples were attached to the side of the casing by using the 300 Omega high temperature cement bond. The cement was chosen for its high thermal conductivity properties, and the low sensitivity to thermal shock it possesses. This type of thermal cement also does not dissolve the insulation surrounding the C01-E thermocouples and creates another insulation barrier for the thermocouples. Thus protecting the thermocouples from breaking and short circuiting during operations. Since the apparatus will be used for gas phase separations, a concern was that solvents or acids could potentially be split on the apparatus thus breaking the thermocouples; the cement that was chosen is resistant to most acids and solvents used for the gas phase separations.

The Omega CN1504-TC multi-zone controller was selected to control the heating zones of the apparatus, and controls the ramp/soak process required for gas phase separations. This control system controls four separate channels and possesses a built in proportional-integral-derivative controller (PID) to measure the feedback from the attached sensors; however, the PID can be shut off if the heaters need to be run continually. The CN1504-TC scans each of the four channels every $0.5 \mathrm{sec}$ to ensure that the temperature at that sensor remains constant. These parts were assembled, and then connected to the top of the GC and to the torch of the ICPMS. Figure 2 shows the completed instrument.

\section{Results}

A thermal analysis of the new instrument was created by using the Matlab R2013B [14] software. After completing the model, the results indicated that at the maximum operating temperature of the quartz column $\left(300^{\circ} \mathrm{C}\right)$ the temperature varied $2.3 \%$ of a degree $\left( \pm 1^{\circ} \mathrm{C}\right)$. Because of the small variation in the temperature down the column, the gas phase separations will be accurate and reproducible.

The NH4.Ho[hfac] 4 and NH4.Er[hfac] 4 were injected via syringe into the GC system, with concentrations of $\sim 4 \mathrm{mg} / \mathrm{mL}$ dissolved in ethyl ether, respectively. The GC was held at a constant temperature of $160^{\circ} \mathrm{C}$ on the entire column. The $\mathrm{NH} 4 \cdot \mathrm{Ho}[\mathrm{hfac}] 4$ and NH4.Er[hfac] 4 was eluted at $3345 \mathrm{~s}$ and $3365 \mathrm{~s}$, respectively, see Figure 3 and Figure 4, with a resolution calculated by Equation (7) [15].

$$
R=\Delta\left(\frac{t_{r}}{w_{\text {avg }}}\right)>1.5
$$

where $t_{r}$ is the retention time of a given peak, $R$ is the separation factor, and 


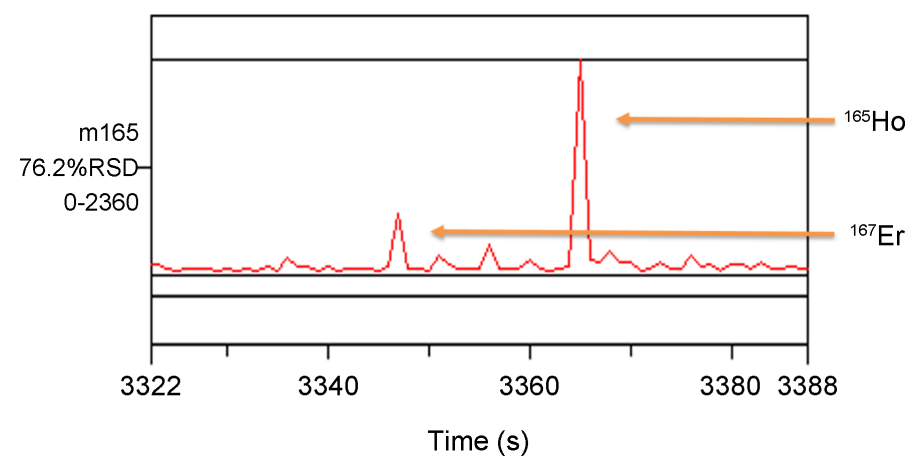

Figure 3. The separation spectrum from the GC-ICP-MS of Ho-165 and Er-167.

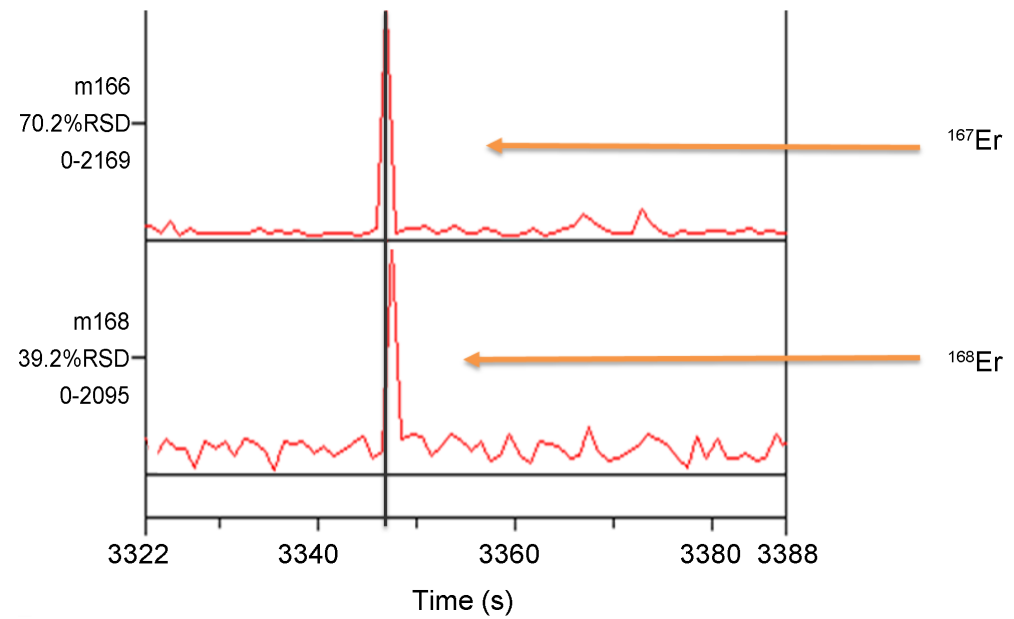

Figure 4. The separation of Er-167 and Er-168.

$w_{\text {avg }}$ is the width of the peak at the base, which is used in Equation (8) to verify the necessary resolution of the sample [16].

$$
\left|\frac{\left(t_{r \mathrm{ro}}-t_{r E r}\right)}{w_{\text {avgHo }}-w_{\text {avgEr }}}\right|>1.5
$$

where $t_{r H o}$ is the retention time for the $\mathrm{NH} 4 \cdot \mathrm{Ho}[\mathrm{hfac}] 4, t_{r E r}$ is the retention time for NH4.Er[hfac] $4, w_{\text {avgHo }}$ is the width at the base of the peak for NH4.Ho[hfac]4, and $w_{\text {avgEr }}$ is the width at the base of the peak for NH4.Er[hfac]4 [17].

The spectra shown in Figure 3 and Figure 4 indicates that the apparatus provides an interface providing excellent medium for separation. Furthermore, the system provides excellent resolution between the compounds that are located in the same group, identical trivalent charge, and similar ionic radius. The separation factor for the new instrument is 8.5, which translates to a separation of $99.9999 \%$. The compounds, for the experimental run were injected into the column at $t=3300 \mathrm{sec}$.

Figure 3 shows the Ho[hfac] 4 compounds that have been separated, and Figure 4 shows the $\operatorname{Er}[\mathrm{Hfac}] 4$ compounds. This shows that there is a separation of $\sim 20$ secs. Also there is a slight separation between the Er-167 and Er-168 isotopes; therefore, the new instrument can effectively analyze isotopic separations as well. 


\section{Conclusion}

By creating this new instrument, analysis of both elemental and isotopic separations can be completed within a 5 minute time span; greatly reducing the time required to perform analysis from the current chemical separation techniques. By adding this capability to the nuclear forensic laboratories this would decrease analysis time and improve elemental and isotropic analysis, thus improving our national response to a nuclear detonation event.

\section{Acknowledgements}

The authors wish to thank Dr. Howard Hall and the Institute for Nuclear Security for their support in performing this work.

\section{Disclosure}

The authors declare that there is no conflict of interest regarding the publication of this article. The authors have no direct financial relationship with any of the equipment manufacturers listed in this publication. The authors have no financial or business interest in any companies that may be affected by the research reported in this paper.

\section{Funding}

This work was performed under grant number DE-NA0001983 from the Stewardship Science Academic Alliances (SSAA) Program of the National Nuclear Security Administration (NNSA). The views expressed are those of the authors and do not necessarily reflect those of the DOE or NNSA.

\section{References}

[1] Skoog, D.A., Holler, F.J. and Crouch, S.R. (2007) Principles of Instrumental Analysis. Sixth Edition, Brooks Cole, Belmont, 1039.

[2] Molgaard, J.J., Auxier, J.D., Oldham, C.J., Cook, M.T., Young, S.A. and Hall, H.L. (2015) Development of Synthetic Nuclear Melt Glass for Forensic Analysis. Journal of Radioanalytical and Nuclear Chemistry, 304, 1293-1301.

[3] Carney, K.P., Finck, M.R., McGrath, C.A., Martin, L.R. and Lewis, R.R. (2013) The Development of Radioactive Glass Surrogates for Fallout Debris. Journal of Radioanalytical and Nuclear Chemistry, 299, 363-372.

[4] Auxier, J.P., Auxier, J.D., Hanson, D. and Hall, H.L. (2014) Modeling Gas Phase of Enthalpy and Entropy of Adsorption and Desorption of $\mathrm{Ln}[\mathrm{Hfac}]_{\mathrm{x}}$ Compounds. 55 th Annual Meeting of the Institute of Nuclear Materials Management, Atlanta, 20-24 July 2014, 1011.

[5] Eichler, R. and Schädel, M. (GSI Darmstadt) (1985) A Monte-Carlo Model of Vacuum Thermochromatography.

[6] Zvara, I. (2008) The Inorganic Radiochemistry of Heavy Elements. Joint Institute for Nuclear Research, Dubna, 247.

[7] Schmidt, F.W., Henderson, R.E. and Wolgemuth, C.H. (1993) Introduction to Thermal Sciences. 2nd Edition, John Wiley \& Sons, Inc., New York.

[8] Hanson, D.E., Garrison, J.R. and Hall, H.L. (2011) Assessing Thermochromatogra- 
phy as a Separation Method for Nuclear Forensics: Current Capability vis-à-vis Forensic Requirements. Journal of Radioanalytical and Nuclear Chemistry, 289, 213223.

[9] Schädel, M. and Shaughnessy, D. (2014) The Chemistry of Super Heavy Elements. Springer, Berlin Heidelberg, 600.

[10] Loveland, W., Morrissey, D.J. and Seaborg, G.T. (2006) Modern Nuclear Chemistry. John Wiley \& Sons Inc., Hoboken, 671.

[11] Auxier, J.D., Stratz, A., Hanson, D., Marsh, M., Jordan, J. and Hall, H.L. Synthesis, Characterization, and Thermogravimetric Analysis of Lanthanide 6,6,7,7,8,8,8-Heptafluoro-2,2-Dimethyl-3,5-Octanedione Complexes for Rapid Separation Applications. Inorganic Chemistry.

[12] Auxier, J.D., Stratz, A., Jordan, J., Hanson, D., Shahbazi, S., Cressy, D., et al. (2016) Thermodynamic Analysis of Volatile Organometallic Fission Products. Journal of Radioanalytical and Nuclear Chemistry, 307, 1621-1627.

[13] Auxier, J.D., Hanson, D., Auxier, J.P., Marsh, M.L., Mull, D.L., Jenkins, D.M., et al. (2014) Gas-Phase Thermochromatographic Separations of Fission and Activation Products. Annual Meeting of the Institute for Nuclear Materials Management, 20-24 July 2014, Atlanta, 9.

[14] Matlab, R. (2013) MATLAB and Statistics Toolbox Release R2013b. The MathWorks, Inc., Massachusetts.

[15] Shahbazi, S., Stratz, S.A., Auxier II, J.D., Hanson, D.E., Marsh, M.L., Hall, H.L. Characterization and Thermogravimetric Analysis of Lanthanide Hexafluoroacetylacetone Chelates. Journal of Radioanalytical and Nuclear Chemistry, 311, 617-626.

[16] Schädel, M. (2013) The Chemistry of Super Heavy Elements.

[17] Loveland, W., Morrissey, D.J. and Seaborg, G.T. (2006) Modern Nuclear Chemistry. John Wiley \& Sons Inc., Hoboken.

\section{Submit or recommend next manuscript to SCIRP and we will provide best service for you:}

Accepting pre-submission inquiries through Email, Facebook, LinkedIn, Twitter, etc. A wide selection of journals (inclusive of 9 subjects, more than 200 journals) Providing 24-hour high-quality service User-friendly online submission system Fair and swift peer-review system Efficient typesetting and proofreading procedure Display of the result of downloads and visits, as well as the number of cited articles Maximum dissemination of your research work

Submit your manuscript at: http://papersubmission.scirp.org/

Or contactwjnst@scirp.org 